\title{
Understanding Skill Acquisition: The Enactive vs. the Ecological Approach and Some Consequences
}

\author{
Alfonsina Scarinzi* \\ (*)Georg-August Universität Göttingen (Germany) \\ Faculty of Philosophy \\ Department of Philology \\ E-mail: alfonsinascarinzi@googlemail.com
}

\begin{abstract}
This theoretical contribution aims at shedding light on the use of the notion of 'enactive' in research on direct perception and on its contribution to the understanding of skill acquisition. While its founders Varela et al. (1991) in the work 'The Embodied Mind' have stressed the specificity of the enactive approach and have insisted on the difference between the enactive and the ecological approach, ecological research tends to use the term 'enactive' as a general umbrella term to indicate intrinsically active perception. This contribution makes the point that the specificity of the notion of 'enactive' cannot be neglected and that this may have some relevant consequences for the understanding and investigation of skill acquisition.
\end{abstract}

\section{Introduction}

In their recent study Araújo \& Davids (2011) propose an ecological definition of skill acquisition. They characterize it as

\begin{abstract}
"the refinement of adaptation processes, achieved by perceiving the key properties of the surrounding layout of the performance environment in the scale of an individual's body and action capabilities. These properties are picked up and used as information to regulate action in specific performance environments (such as the surface texture of ice and blades for the triple salto)" (Araújo \& Davids, 2011:19).
\end{abstract}

The relevant implication of this definition is that it rejects the cognitive psychological view, according to which skill acquisition is the establishment of an internal state or representation of an act which is believed to be acquired as a result of learning and task experience and stored somewhere. On the other hand, the weak point of the mentioned definition consists in the fact that against the background of the state-of-theart in research on the ecological approach to direct perception, it is formulated in a way that does not allow to recognize the specifically ecological nature of the definition they propose, for the authors in their study refer to theoretical premises such as 'the possibilities and capabilities of action of the organism's body', which the ecological approach to direct perception shares with the so called enactive approach developed by Varela et al. (1991). Without making some specific observations, which will be formulated in the following sections, the very ecological nature of the definition can be easily equated with the enactive approach, for some common tenets the two approaches share have led the ecological research to present the ecological approach as an articulation of the enactive approach [5,6], as Mossio and Taraborelli (2008) observe.

The aim of the present contribution is to discuss the question whether the two approaches can be considered to be two sides of the same coin or whether they are two completely different ways of looking at direct perception, as Mossio and Taraborelli (2008) and especially Varela et al. (1991) claim. This contribution aims at shedding light on how a positive answer to both questions can affect the understanding of skill acquisition.

\section{The Enactive in the Ecological Approach and Skill Acquisition}

The notion of 'enactive' has been adopted in the ecological approach to perception as an umbrella term to describe the linkage that exists between how we move and what we perceive $[5,6]$. At this broad general theoretical level the 'enactive' of the ecological approach consists in the fact that the perceptual guidance of action and the resultant emergence of cognition are possible because properties of the

This is an Open Access article distributed under the terms of the Creative Commons Attribution-Noncommercial License 3.0, which permits unrestricted use, distribution, and reproduction in any noncommercial medium, provided the original work is properly cited. 
animal-environment system are specified in potential sensory stimulation where specification consists of lawful relations [5]. According to this way of looking at direct perception, the 'enactive' is linked to the Gibsonian notion of affordances, that is to the possible actions in any given situation. The use of 'enactive' in the ecological approach stresses the view that affordances are perceived in a direct way rather than derived through cognitive operations.

This way of presenting the notion of 'enactive' seems to be a way to paraphrase the ecological animalenvironment relation and the linkage between how the animal moves and what the animal perceives. Nothing more. Against this background, the definition of skill acquisition by Araújo \& Davids (2011) mentioned above can be considered to be both ecological and enactive. But, again, in this way the specificity of the ecological and of the enactive approach disappears. This does not correspond to what the founders of the enactive approach Varela et al. (1991) had in mind. They stressed the fundamental difference between the ecological and the enactive approach and hence the specificity both of the enactive and of the ecological approach to human perception. Putting this difference into the foreground will help to acknowledge in a more explicit way the ecological specificity of the skill acquisition definition mentioned above and how the enactive approach can contribute to understanding the phenomenon of skill acquisition in a different, alternative way.

\section{The Enactive in the Enactive Approach and Skill Acquisition}

In Chapter 9 of The Embodied Mind Varela et al. (1991:203-204) clearly state the reasons why the ecological approach by Gibson cannot be completely combined with their enactive approach. They make the point that the ecological approach is a monistic environment-centric system, while the enactive approach is a middle-way, designed to be an alternative to monism and to dualism. According to the authors, the point that one can easily miss and that represents the difference between the ecological and the enactive approach is that in the ecological approach perceptually guided action consists in picking up invariances in the ambient light. In Gibson's approach these invariances as well as the environmental properties they specify do not depend on the perceptually guided activity of the animal. Invariances are there to be discovered. An affordance is always there to be perceived. In this sense it cannot be 'enactive', because it cannot be brought forth by the animal in a relation of co-determination with the environment. It can just be picked up. Varela et al. (1991:203) briefly summarize that in their enactive approach the environment is not independent. Rather, it is enacted by histories of coupling. While Gibson claims that perception is direct detection, Varela et al. (1991) claim that it is sensorimotor enactment. They point out that they do not build up the theory of perception from the environment as Gibson does. Rather, they build it up from the structural coupling of the animal by specifying the sensorimotor patterns that enable action to be perceptually guided. Moreover, Varela et al. (1991) criticize the mistaken assumption of Gibsonian researchers that animal-environment mutuality is sufficient for direct perception. They point out that from mutuality does not follow that the act of perceiving is direct in the Gibsonian sense of responding to invariants. Mossio \& Taraborelli (2008) have illustrated the difference of the two approaches by pointing out that in contrast to ecological approaches, the enactive approach does not characterize perceptual invariants as properties of the sensory stimulation revealed through motion. A perceptual invariant in the enactive approach is a property of sensorimotor couplings that remains invariant throughout transformations. Whenever a sensory transformation co-occurs with a movement, those properties that remain unchanged in this coupling constitute a sensorimotor invariant. This means that movement has a constitutive role in the determination of sensorimotor invariants in the enactive approach, whereas it is instrumental in the determination of invariants in the ecological approach.

Against this background, understanding skill acquisition according to the enactive approach implies the study of the refinement of adaptation processes, achieved by bringing forth these key processes in a history of structural coupling with the environment.

\section{Summing Up: Some Consequences for the Understanding of Skill Acquisition}

The consequence of making explicit the difference between the ecological and the enactive approach for the understanding of skill acquisition leads to acknowledging the ecological specificity of the skill acquisition definition proposed by Araújo \& Davids (2011). Against the background of the arguments of the founders of the enactive approach, it seems implausible and confusing to use the notion of 'enactive' to characterize the ecological approach. Putting into focus the differences of the enactive and of the ecological approach implies acknowledging the different point of view that characterizes both approaches. The ecological approach is an environment-centric 
approach, while the enactive approach is a relationcentric approach.

An enactive definition of skill acquisition as an alternative to the ecological definition should not focus on the perception of the key properties of the surrounding layout of the performance environment in the scale of an individual's body and action capabilities, which are picked up by the animal. Rather, it should focus on the way the animal in the refinement of adaptation processes in skill acquisition according to the animal's structure brings forth or enacts such properties in its relation of co-determination with the environment.

\section{Providing Examples: The Field of Computer Games}

The following examples may better illustrate the difference between ecological skill acquisition and enactive skill acquisition. In the following I will draw the attention to skill acquisition in technologically mediated interactions in the field of computer games.

The game Timesplitter 2 discussed in [2] allows to highlight the environment-centric nature of the ecological skill acquisition and its instrumental role of movement. Timesplitter 2 is a split-screen multiplayer focused first-person shooter. Many people can play with or against each other on the same television set. In the game there are two kinds of crates, exploding and non-exploding ones. In the way the player learn to discriminate and test out the affordances by shooting and seeing hence what happens, the environment centric nature of the ecological skill acquisition becomes visible. In order to find out the affordances of the virtual object the subject has to accomplish an instrumental movement. After testing the player becomes aware of the fact that in the game crates often can be open if you fire at them and inside the player can find different items. The skill acquisition is ecological in the sense of [1], for the properties of the virtual object are picked up and used as information to regulate action in the performance game environment. In order to do justice to ecological skill acquisition process as an adaptation process game designers need just to focus on the affordance of the virtual object a player can pick up while learning to play the game.

A good example for the enactive skill acquisition is provided by the range of full-body games one can play using Kinect (http://www.xbox.com/fr-FR/Kinect). Kinect is an action driven interface that is capable of conveying and understanding the gestures of the user, in order to provide an adequate response in perceptual terms. Kinect is conceived for the Xbox 360 video game console. It is based around a webcam-style add- on peripheral for the Xbox 360 console and enables users to control and interact with the Xbox 360 without the need to touch a game controller, through a natural user interface using gestures, skilled movements and spoken commands. It tracks the player's whole body and integrates this body state information into the game. The technologically mediated world comes into being thanks to the player's sensorimotor coupling with the technologically mediated environment. Skill acquisition in this sort of context means for the subject finding out sensorimotor strategies according to the bodily structure of the player that allows to bring forth a viable game situation. This means that movement has a constitutive role in the determination of sensorimotor invariants in this sort of game. Consequently, a child, an adult, elderly people, persons with motor impairment or sportsmen would bring forth different sensorimotor invariants and game situations according to their bodily structure. Each enacted environment will be a viable one according to the structure of the player. The attention of the player is not focused on the affordances of an object. Rather, it is focused on the determination of the sensorimotor relation with the environment movement is constitutive of.

The focus on the relation of co-determination between the perceiver and the mediated environment in skill acquisition is the main reason why Kinect technology is used especially in serious games for motor rehabilitation, e. g. in the motor rehabilitation of chronic back pain people [4]. Instead of having to accomplish instrumental movements to pick up the affordances of the environment, the player-patient is motivated to accomplish skilled movements to create and bring forth an own viable game situation. In order to do justice to enactive skill acquisition process as an adaptation process game designers need to focus on the wide range of viable enactments human sensorimotor couplings with the mediated environment may generate. Not affordance but viability is crucial for enactive skill acquisition.

\section{Conclusion and Future Work}

Even if the ecological and the enactive approach share common elements and at a broad general theoretical level they can be considered to be two sides of the same coin - being the 'same coin' the study of direct perception - , their difference is fundamental and cannot be left aside. Taking it into account in the study and understanding of skill acquisition means to make a decision: to focus the attention either on the environment and its invariance, which is ecological, or on the relation of co-determination between the environment and the animal, which according to the 
founders of enactivism is enactive and not ecological in the Gibsonian sense.

The challenge that follows for future studies on skill acquisition implies finding a way to make visible the mentioned difference at the empirical level of the investigation of skill acquisition and to underpin the enactive claim with strong and convincing empirical results.

\section{References}

[1] Araújo, D. and Davids, K. What Exactly is Acquired During Skill Acquisition?, Journal of Consciousness Studies, 18, pages $7-23,2011$

[2] Linderoth, J. and Bennerstedt, U. This is not a Door: an Ecological Approach to Computer Games, Situated Play, Proceedings of DiGRA Conference, pages $600-$ 609, 2007
[3] Mossio, M. and Taraborelli, D. Action-dependent Perceptual invariants: from ecological to sensorimotor approaches, Consciousness and Cognition, Elsevier, pages $1324-1340,2008$

[4] Schoenauer, C., Pintaric, T. and Kaufmann, H. Full Body Interaction for Serious Games in Motor Rehabilitation, $A H$ '11, Mar 12-14, 2011

[5] Stoffregen, T., Bardy, B. and Mantel, B. Affordances in the design of enactive systems, Virtual Reality, Springer London, pages 4 - 10, May 2006

[6] Stoffregen, T. A. and Bardy, B. G. Multisensory enactive interfaces and the global array, http://www.interdisciplines.org/enaction/papers/3, 2004

[7] Varela, F., Thompson, E. and Rosch, E. The Embodied Mind, The MIT Press, 1991 